\title{
GENERALIZED CR-SUBMANIFOLDS OF THE TRANS HYPERBOLIC SASAKIAN MANIFOLD
}

\begin{abstract}
The purpose of the present paper is to study generalized CR- submanifolds of the trans hyperbolic Sasakian manifold and obtained their basic properties. The conditions under which the distribution of generalized CR-submanifolds of the trans hyperbolic Sasakian manifold is integrable have been obtained. Totally geodesic generalized CR- submanifolds of the trans hyperbolic Sasakian manifold have also been studied.
\end{abstract}

\section{Introductution and preliminaries}

Bejancu defined and studied CR-submanifolds of Kaehlerian manifolds [1]. CR-submanifolds of Sasakian manifolds were studied by Kobayashi [8]. Bejancu and Papaghuic defined almost semi - invariant submanifold of Sasakian manifold [3]. Oubina studied a new class of almost contact Riemannian manifold known as Trans Sasakian manifold [6], which generalizes both $\alpha$-Sasakian and $\beta$-Kenmotsu structure. Ion Mihai introduced a new class of submanifolds called genealised CR-submanifolds of Sasakian manifolds [4]. M. D. Upadhyay studied an almost contact hyperbolic $(f, g, \eta, \xi)$-structure [9]. Bhatt and Dube studied on a CR-submanifolds of a trans hyperbolic Sasakian manifold [7]. Submanifolds of hyperbolic contact metric manifold were studied by R. B. Pal [11]. N. K. Joshi and K. K. Dube studied a semi invariant submanifold of an almost r-contact hyperbolic metric manifold [10]. The purpose of the present paper is to study the generalised CR-submanifold of a trans Hyperbolic Sasakian manifold.

Let $V_{n}$ be an n-dimensional almost hyperbolic contact metric manifold with the almost hyperbolic contact metric structure $(F, U, u, g)$, where $F$ is a tensor field of type $(1,1), U$ is a vector field, $u$ is a 1 -form and $g$ is the Riemannian metric on $V_{n}$. Then the following conditions [9] are satisfied:

Classification number of my paper is: $57-\mathrm{XX}$-manifolds and cell complexes or 58A05Differentiable manifolds, foundations.

Key words and phrases: Submanifolds, Cosymplectic manifolds, Hyperbolic Sasakian manifolds. 


$$
\begin{gathered}
F^{2} X=X+u(X) U, \quad F U=0, \quad u \circ F=0, \quad u(U)=-1, \\
g(F X, F Y)=-g(X, Y)-u(X) u(Y) \\
g(F X, Y)=-g(X, F Y)
\end{gathered}
$$

for vector fields $X, Y$ tangent to $V_{n}$. An almost hyperbolic contact metric structure $(F, U, u, g)$ on $V_{n}$ is called trans hyperbolic Sasakian [7] if and only if

$$
\left(\bar{\nabla}_{X} F\right) Y=\alpha\{g(X, Y) U-u(Y) F X\}+\beta\{g(F X, Y) U-u(Y) F X\},
$$

for all $X, Y$ tangent to $V_{n}$ and $\alpha, \beta$ non zero constant. Where $\nabla$ is the Riemannian connexion with respect to $g$. From (1.4) it follows that

$$
\bar{\nabla}_{X} U=-\alpha F X+\beta(X-u(X) U),
$$

for any vector field $X$ tangent to $V_{n}$. Now if $V_{m}$ be an $m$-dimensional submanifold isometrically immersed in a trans hyperbolic Sasakian manifold $V_{n}$ such that the structure vector field $U$ of $V_{n}$ is tangent to the submanifold $V_{m}$. We denote by $\{U\}$ the 1-dimensional distribution spanned by $U$ on $V_{m}$ and $\{U\}^{\perp}$ the complementary orthogonal distribution to $\{U\}$ in $T\left(V_{m}\right)$. For any $X \in T\left(V_{m}\right)$ we have $g(F X, U)=0$. Then we let us put,

$$
F X=b X+c X
$$

where $b X \in\{U\}^{\perp}$ and $c X \in T^{\perp}\left(V_{m}\right)$. Thus $X \rightarrow b X$ is an endomorphism of the tangent bundle $T\left(V_{m}\right)$ and $X \rightarrow c X$ is a normal bundle valued 1-form on $V_{m}$.

1.1. Definition. A submanifold $V_{m}$ of an almost hyperbolic contact metric manifold $V_{n}$ with almost hyperbolic contact metric structure $(F, U, u, g)$ is said to be generalized CR-submanifold if $D_{X}^{\perp}=T_{X}\left(V_{m}\right) \cap F T_{X}^{\perp}\left(V_{m}\right) ; x \in V_{m}$ defines a differentiable subbundle of $T_{x}\left(V_{m}\right)$. Thus for $X \in D^{\perp}$ one has $b X=0$. We denote by $\mathrm{D}$ the complementary orthogonal subbundle to $D^{\perp} \oplus\{U\} \operatorname{in} T\left(V_{m}\right)$.

For any $X \in D, b X \neq 0$. Also we have $\mathrm{bD}=\mathrm{D}$. Thus for generalised CR-submanifold $V_{m}$ we have the orthogonal decomposition

$$
T\left(V_{m}\right)=D \oplus D^{\perp} \oplus\{U\} .
$$

\section{Lemmas}

For generalized CR-submanifold $V_{m}$ of the trans hyperbolic Sasakian manifold $V_{n}$, we denote by $\mathrm{g}$ both the Riemannian metrics on $V_{n}$ and $V_{m}$.

For each $X \in T\left(V_{m}\right)$ we put

$$
X=P X+Q X-u(X) U
$$


where $P X$ and $Q X$ belong to the distribution $D$ and $D^{\perp}$ respectively. For any $N \in \frac{\perp}{X}\left(V_{m}\right)$ we put

$$
N=t N+f N
$$

where $t N$ is tangential part of $F N$ and $f N$ is the normal part of $F N$. By using (1.2) we have

$$
g(F X, c Y)=g(F X, b Y+c Y)=g(F X, F Y)=-g(X, Y)=0,
$$

for $X \in D_{X}^{\frac{1}{X}}$ and $Y \in D_{X}$. Therefore,

$$
g\left(F D_{X}^{\perp}, c D_{X}\right)=0 \text {. }
$$

We denote by $v$ the orthogonal complementary vector bundle to $F D^{\perp} \oplus$ $c \operatorname{DinT} T^{\perp}\left(V_{m}\right)$. Thus we have

$$
T^{\perp}\left(V_{m}\right)=F D^{\perp} \oplus c D \oplus v .
$$

2.1. LEMMA. The morphism $t$ and $f$ satisfy

$$
t\left(f D^{\perp}\right)=D^{\perp}
$$

and

$$
t(c D) \subset D \text {. }
$$

Proof. For $X \in D^{\perp}$ and $Y \in D, g(t F X, Y)=g(t F X+f F Y, Y)=$ $g\left(F^{2} X, Y\right)=g(X, Y)=0$.

Also $g(t F X, U)=g\left(F^{2} X, U\right)=g(F X, U)=0$. Therefore $t\left(f D^{\perp}\right) \subset D^{\perp}$. For $X \varepsilon D^{\perp}$, we have, $X=F^{2} X=t F X+f F X$ which implies $X=t F X$.

Consequently, $D^{\perp} \subset t\left(F D^{\perp}\right)$. Hence the relation (2.5) follows. The relation (2.6) is trivial.

Now we denote by $\bar{\nabla}$ (resp. $\nabla$ ) the Riemannian connexion on $V_{n}$ (resp. $V_{m}$ ) with respect to the Riemannian metric $g$. The linear connection induced by $\bar{\nabla}$ on the normal bundle $T^{\perp}\left(V_{m}\right)$ is denoted by $\nabla^{\perp}$. Then the equation of Guass and Wiengarten are given by

$$
\begin{aligned}
& \bar{\nabla}_{X} Y=\nabla_{X} Y+h(X, Y), \\
& \bar{\nabla}_{X} N=-A_{N} X+\nabla_{X}^{\perp} N,
\end{aligned}
$$

for $X, Y \in T\left(V_{m}\right)$ and $N \in T^{\perp}\left(V_{m}\right)$. Where $h$ is the second fundamental form of $V_{m}$ and $A_{N}$ is the fundamental tensor of Wiengarten with respect to the normal section $N$. These tensor fields are related by

$$
g(h(X, Y), N)=g\left(A_{N} X, Y\right),
$$

for $X, Y \in T\left(V_{m}\right)$ and $N \in T^{\perp}\left(V_{m}\right)$.

We denote

$$
W(X, Y)=\nabla_{X} b P Y-A_{C P Y} X-A_{F Q Y} X
$$


2.2. LEMMA. Let $V_{m}$ be a generalized CR-submanifold of the trans hyperbolic Sasakian manifold $V_{n}$, then we have

(2.10) $P(W(X, Y))-b P \nabla_{X} Y-P \operatorname{th}(X, Y)=-\alpha u(Y) P X-\beta u(Y) P b X$

(2.11) $Q(W(X, Y))-Q \operatorname{th}(X, Y)=-\alpha u(Y) Q X-\beta u(Y) Q b X$

(2.12) $u(W(X, Y))=\alpha g(F X, F Y)+\beta g(F b X, F b Y)$

(2.13) $h(X, b P Y)+\nabla_{X}^{1} c P Y+\nabla_{X}^{\frac{1}{X}} f Q Y-c P \nabla_{X} Y-f h(X, Y)=-\beta u(Y) c X$

for $X, Y \in T\left(V_{m}\right)$.

Proof. Using (1.6), (2.1), (2.2), (2.7) and (2.8) in (1.4) we have, for $X, Y \in$ $T\left(V_{m}\right)$

$\bar{\nabla}_{X} F Y-\bar{\nabla}_{X} F Y=\alpha\{g(X, Y) U-u(Y) F X\}+\beta\{g(F X, Y) U-u(Y) F X\}$, $\bar{\nabla}_{X} F P Y+\bar{\nabla}_{X} F Q Y-F \nabla_{X} Y-f h(X, Y)$

$$
=\alpha\{g(X, Y) U-u(Y) F X\}+\beta\{g(F X, Y) U-u(Y) F X\},
$$

$\nabla_{X} b P Y+h(X, b P Y)-A_{c P Y} X+\nabla \frac{1}{X} c P Y-A_{F Q Y} X$

$+\nabla \frac{1}{X} F Q Y-b \nabla_{X} Y-c \nabla_{X} Y-F P h(X, Y)-F Q h(X, Y)$

$=\alpha\{g(X, Y) U-u(Y) F X\}+\beta\{g(F X, Y) U-(Y) F X\}$,

$\nabla_{X} b P Y+h(X, b P Y)-A_{c P Y} X+\nabla_{X}^{1} c P Y-A_{F Q Y} X+\nabla \frac{1}{X} F Q Y-b P \nabla_{X} Y$

$-c Q \nabla_{X} Y-Q b \nabla_{X} Y-c P \nabla_{X} Y-P t h(X, Y)$

$-P f h(X, Y)-Q \operatorname{th}(X, Y)-Q f h(X, Y)$

$=\alpha\{g(X, Y) U-u(Y) F X\}+\beta\{g(F X, Y) U-u(Y) F X\}$,

$\nabla_{X} b P Y+h(X, b P Y)-A_{c P Y} X+\nabla_{X}^{\frac{1}{X}} c P Y-A_{F Q Y} X+\nabla_{X}^{\frac{1}{X}} F Q Y$

$-b P \nabla_{X} Y-c P \nabla_{X} Y-F Q \nabla_{X} Y-P \operatorname{th}(X, Y)-f h(X, Y)-Q t h(X, Y)$

$=\alpha\{g(X, Y) U-(Y) F X\}+\beta\{g(F X, Y) U-u(Y) F X\}$.

Taking components on each of the vector bundle $D, D^{\perp},\{U\}$ and $T^{\perp}\left(V_{m}\right)$ respectively, we get the required results.

2.3. LemMA. For generalized CR-submanifolds $V_{m}$ of the trans hyperbolic Sasakian manifold $V_{n}$, the following relations holds:

$$
\begin{aligned}
& P\left(t \nabla \frac{1}{X} N+A_{f N} X-\nabla_{X} t N\right)=b P A_{N} X, \\
& Q\left(t \nabla \frac{1}{X} N+A_{f N} X-\nabla_{X} t N\right)=0, \\
& u\left(A_{f N} X-\nabla_{X} t N\right)=-\beta g(c X, N), \\
& h(X, t N)+F Q A_{N} X+\nabla_{X}^{\frac{1}{X}} f N+c P A_{N} X=f \nabla_{X}^{\frac{1}{X}} N,
\end{aligned}
$$

for $X \in T\left(V_{m}\right)$ and $N \in T^{\perp}\left(V_{m}\right)$.

Proof. For $X \in T\left(V_{m}\right)$ and $N \in T^{\perp}\left(V_{m}\right)$ by using (1.6), (2.1), (2.2) and $(2.7),(2.8)$ in (1.4) we get, 


$$
\begin{aligned}
& \left(\bar{\nabla}_{X} F\right) Y=\alpha\{g(X, Y) U-u(Y) F X\}+\beta\{g(F X, Y) U-u(Y) F X\} \\
& \bar{\nabla}_{X} F Y-F \bar{\nabla}_{X} Y=\beta g(c X, N) U, \\
& \nabla_{X} F Y+h(X, F Y)-F \bar{\nabla}_{X}(P Y+Q Y-u(Y) U)=\beta g(c X, N) U \\
& \nabla_{X} t N+h(X, t N)+\nabla_{X} f N+h(X, f N)-F P \bar{\nabla}_{X} Y-F Q \bar{\nabla}_{X} Y=\beta g(c X, N) U \\
& P \nabla_{X} t N+Q \nabla_{X} t N-u\left(\nabla_{X} t N\right) U+h(X, t N)-P A_{f N} X-Q A_{f N} X-u\left(A_{f N} X\right) U \\
& \quad+\nabla \frac{1}{X} f N+b P A_{N} X-f P \nabla_{X}^{\perp} N+c P A_{N} X-F Q \nabla_{X}^{\perp} N+F Q A_{N} X \\
& \quad=\beta g(c X, N) U, \\
& P \nabla_{X} t N+Q \nabla_{X} t N-u\left(\nabla_{X} t N\right) U+h(X, t N)-P A_{f N} X-Q A_{f N} X \\
& \quad-u\left(A_{f N} X\right) U+\nabla_{X}^{\frac{1}{X}} f N+b P A_{N} X-P t \nabla \frac{1}{X} N-Q t \nabla \frac{1}{X} N+c P A_{N} X \\
& \quad-f \nabla \frac{1}{X} N+F Q A_{N} X=\beta g(c X, N) U .
\end{aligned}
$$

Taking components on each of the vector bundles $D, D^{\perp},\{U\}$ and $T^{\perp}\left(V_{m}\right)$ respectively, we get the required results.

2.4. LEMMA. For generalized CR-submanifold $V_{m}$ of the trans hyperbolic Sasakian manifold $V_{n}$, we have the following:

$$
\begin{aligned}
& \nabla_{X} U=-\alpha b X+\beta X ; h(X, U)=-\alpha c X \text { for } X \in D, \\
& \nabla_{Y} U=\beta Y ; h(Y, U)=-\alpha F Y, \text { for } Y \in D^{\perp}, \\
& \nabla_{U} U=0 ; h(U, U)=0 .
\end{aligned}
$$

Proof. The proof follows from (1.5) by using (1.6) (2.1) and (2.7), we have

$$
\begin{aligned}
& \bar{\nabla}_{X} U=-\alpha b X-\alpha c X+\beta X, \\
& \nabla_{X} U+h(X, U)=-\alpha b X-\alpha c X+\beta X, \\
& \nabla_{X} U=-\alpha b X+\beta X, \quad h(X, U)=-\alpha c X .
\end{aligned}
$$

Hence (2.18) follows.

$$
\begin{aligned}
& \bar{\nabla}_{Y} U=-\alpha F Y+\beta(Y-u(Y) U, \\
& \nabla_{Y} U+h(Y, U)=-\alpha F Y+\beta Y-u(Y) \beta U, \\
& \nabla_{Y} U=\beta Y ; \quad h(Y, U)=-\alpha F Y, \quad \text { for } Y \in D^{\perp} .
\end{aligned}
$$

Hence (2.19) follows.

$$
\begin{aligned}
& \bar{\nabla}_{U} U=-\alpha F U+\beta(U-u(X) U), \\
& \nabla_{U} U+h(U, U)=-\alpha F U+\beta(U-u(X) U), \\
& \nabla_{U} U=0, \quad h(U, U)=0 .
\end{aligned}
$$

Hence (2.20) follows.

2.5. LEMMA. Let $V_{m}$ be a generalized $C R$-submanifold of the trans hyperbolic Sasakian manifold $V_{n}$. We have the following:

$$
A_{F X} Y=A_{F Y} X, \quad \text { for } X, Y \in D^{\perp} \text {. }
$$


Proof. By using (1.3), (1.4), (2.7) and (2.9) we get

$$
\begin{aligned}
& g\left(A_{F X} Y, Z\right)=g(h(Y, Z) F X)=g\left(\bar{\nabla}_{Z} Y, F X\right)=g\left(F \bar{\nabla}_{Z} Y, X\right), \\
& =g\left(\bar{\nabla}_{Z} F Y, X\right)=g\left(F Y, \bar{\nabla}_{Z} X\right)=g(h(Z, X), F Y) \\
& =g(h(X, Z), F Y)=g\left(A_{F Y} X, Z\right)
\end{aligned}
$$

for $X, Y \in D^{\perp}$ and $Z \in T\left(V_{m}\right)$. Hence the lemma follows.

2.6. LEMMA. Let $V_{m}$ be a generalized CR-submanifold of the trans hyperbolic Sasakian manifold. We have the following:

$V \notin D^{\perp}, \quad$ for $V \in D^{\perp}$

$\nabla_{U} S \notin D, \quad$ for $S \in D$.

Proof. Let us take $X=U$ and $V=F N$ in (2.14) where $N \in F D$. Taking account that $t N=F N, f N=0$ we get $P t \nabla \frac{1}{U} N-P \nabla_{U} V=b P A_{N} U$

$$
P t \nabla \frac{1}{U} N-b P A_{N} U=P A_{U} V .
$$

The second relation of (2.18) gives $g\left(P N_{N} U, S\right)=g\left(A_{N} U, S\right)=g(h(S, U) N)=-\alpha g(c S, N)=0, \quad$ for $S \in D$. Hence (2.24) becomes

$$
P t \nabla_{U}^{\perp} N=P \nabla_{U} V
$$

On the other hand (2.17) implies

$$
h(U, V)=f \nabla \frac{1}{U} N-F Q A_{N} U
$$

for $V \in D^{\perp}, h(U, V)=h(V, U)=-\alpha F V \in F D^{\perp}$ by (2.19).

Now for $X \in D^{\perp}$, by virtue of Lemma (2.5) and of equation (2.9) we have

$$
\begin{aligned}
& g(h(U, V), F X)=g(h(V, U), F X)=g\left(A_{F X} V, U\right)=g\left(A_{F V} X, U\right), \\
& =g(h(X, U), F V)=g(h(X, U), N)=g\left(A_{N} U, X\right)=g\left(F A_{N} U, F X\right), \\
& =g\left(F P A_{N} U, F X\right)+g\left(F Q A_{N} U, F X\right)=g\left(F Q A_{N} U, F X\right),
\end{aligned}
$$

since $c D^{\perp} \in F^{\perp}$.

Therefore $h(U, V)=F Q A_{N} U$, which together with (2.26) implies $f \nabla \frac{\perp}{U} N$ $=2 F Q A_{N} U$, hence $\nabla \frac{1}{U} N \notin F D^{\perp}$, since $F$ is an automorphism of $c D \oplus v$. Thus $t \nabla \frac{1}{U} N \notin D^{\perp}$ and from (2.25) it follows that

$$
P \nabla_{U} V \neq 0, \quad \text { for all } V \in D^{\perp} \text {. }
$$

Next from (2.16) we have

$$
u\left(\nabla_{U} V\right) \neq 0
$$

for all $V=F N \in D^{\perp}$, where $N \in F D^{\perp}$.

Hence (2.22) follows from (2.27) and (2.28). 
Finally by using (2.1), (2.20) and (2.22), we have

$$
g\left(\nabla_{U} S, X\right) \neq g\left(\nabla_{U} S, P X\right) \quad \text { for } X \in T V_{m}
$$

and $S \in D$. Thus we have $\nabla_{U} S \notin D$, for $S \in D$ and this completes the proof.

\section{Integrability of distributions}

3.1. Theorem. For generalized CR-submanifold $V_{m}$ of the trans hyperbolic Sasakian manifold $V_{n}$, the distribution $D^{\perp}$ is always involutive.

Proof. For $X, Y \in D^{\perp}$ by using (2.19) we get,

$$
\begin{aligned}
g([X, Y], U) & =g\left(\nabla_{X} Y, U\right)-g\left(\nabla_{Y} X, U\right) \\
& =g\left(X, \nabla_{Y} U\right)-g\left(Y, \nabla_{X} U\right)=0 .
\end{aligned}
$$

On the other hand from (2.10) we have

$$
b P \nabla_{X} Y=-P A_{F Y} X-P \operatorname{th}(X, Y), \quad \text { for } X, Y \in D^{\perp}
$$

and then using lemma (2.5) we get from (3.2)

$$
b P[X, Y]=0, \quad \text { for } X, Y \in D^{\perp} .
$$

As $\mathrm{b}$ is an automorphism of $\mathrm{D}$, the theorem follows from (3.1) and (3.3).

3.2. THEOREM. For generalized CR-submanifold $V_{m}$ of the trans hyperbolic Sasakian manifold $V_{n}$, the distribution $D$ is never involutive.

Proof. For $X, Y \in D$ by using (2.18), we have

$$
\begin{aligned}
g([X, Y], U) & =g\left(\nabla_{X} Y, U\right)-g\left(\nabla_{Y} X, U\right)=g\left(X, \nabla_{Y} U\right)-g\left(Y, \nabla_{X} U\right) \\
& =-\alpha g(X, b Y)+\alpha g(Y, B X)=2 \alpha g(Y, b X) .
\end{aligned}
$$

Taking $X \neq 0$ and $Y=b X$ in (3.4), it follows that $\mathrm{D}$ is never involutive.

3.3. THEOREM. For generalized CR-submanifold $V_{m}$ of the trans hyperbolic Sasakian manifold $V_{n}$, the distrbution $D^{\perp}$ is totally geodesic in $V_{m}$ iff

(3.5) $h(X, b Z)+\nabla_{X}^{\perp} c Z \in c D \oplus v \quad$ for $\quad X \in D^{\perp} \quad$ and $\quad Z \in D \oplus\{U\}$. Proof. For $X, Y \in D^{\perp}, Z \in D \oplus\{U\}$, and using (1.3), (1.4), (2.7)and (2.8), we get

$$
\begin{aligned}
& g\left(\nabla_{X} Y, Z\right)=-g\left(Y, \bar{\nabla}_{X} Z\right)=-g\left(\bar{\nabla}_{X} Z, Y\right)=-g\left(F \bar{\nabla}_{X} Z, F Y\right) \\
& =-g\left(\bar{\nabla}_{X} b Z+\bar{\nabla}_{X} c Z, F Y\right) \\
& =-g\left(\nabla_{X} b Z+h(X, b Z)-A_{c Z} X+\nabla_{X}^{\perp} c Z, F Y\right) \\
& g\left(\nabla_{X} Y, Z\right)=-g\left(h(X, b Z)+\nabla_{X}^{\perp} c Z, F Y\right) .
\end{aligned}
$$

Hence the theorem follows from (3.6).

3.4. TheOREM. Let $V_{m}$ be a generalized CR-submanifold of the trans hyperbolic Sasakian manifold $V_{n}$. Then the distribution $D \oplus\{U\}$ is involutive 
and totally geodesic in $V_{m}$ if and only if,

$$
h(X, b Y)+\nabla \frac{1}{X} c Y \in c D \oplus v \text { for } X, Y \in D \oplus\{U\} .
$$

Proof. For $X, Y \in D \oplus\{U\}$ and $Z \in D^{\perp}$ by using (1.3), (1.4), (1.6), (2.7) and (2.8), we obtain

$$
\begin{aligned}
& g\left(\nabla_{X} Y, Z\right)=g\left(\bar{\nabla}_{X} Y, Z\right)=g\left(F \bar{\nabla}_{X} Y, F Z\right), \\
& g\left(\nabla_{X} Y, Z\right)=g\left(\nabla_{X} b Y+h(X, b Y)-A_{c Y} X+\nabla_{X}^{1} c Y, F Z\right) .
\end{aligned}
$$

Hence the theorem follows from (3.8).

Acknowledgements. The authors are thankful to the referee for his valuable suggestions and modifications.

\section{References}

[1] A. Bejancu, CR-submanifold of a Kaehler manifold, I, Proc. Amer. Math. Soc. 69 (1978), 135-42.

[2] A. Bejancu, CR-submanifold of a Kaehler manifold II, Trans. Amer. Math. Soc. 250 (1979), 331-45.

[3] A. Bejancu and N. Papaghuic, An almost semi-invariant submanifold of Sasakian manifold, Bull. Math. Roumanie, Tome 28 (76) (1984), nr. 1, 13-18.

[4] I. Mihai, Geometry and Topology of Submanifolds, Vol. VII, World Scientific, Singapore, (1995), 186-88.

[5] I. Mihai, Geometry and Topology of Submanifolds, Vol. VIII, World Scientific Singapore, (1996), 265-68.

[6] J.A. Oubina, New classes of almost contact metric structures, Publ. Math. Debrecen 32 (1985), 187-93.

[7] L. B hatt and K. K. Dube, On CR-submanifold of trans hyperbolic Sasakian manifold, Acta Cienc. Indica 29 No. 1, (2003), 91-96.

[8] M. Kobayashi, CR-submanifold of a Sasakian manifold, Tensor N.S. 35 (1981), 297-307.

[9] M. D. Upadhyay and K. K. Dube, Almost contact hyperbolic $(f, g, \eta, \xi)$-structure, Acta Math. Acad. Scient. Hung. 28 (1976), 13-15.

[10] N. K. Joshi and K. K. Dube, Semi invariant submanifold of an almost r-contact hyperbolic metric manifold, Demonstratio Math. 34, no. 1 (2001), 135-143.

[11] R. B. Pal, Submanifolds of hyperbolic contact metric manifold, Acta Cienc. Indica Math. 22 (1996), 399-404.

DEPARTMENT OF MATHEMATICS

STATISTICS AND COMPUTER SCIENCE

CBSH, G.B.PANT UNIV.OF AG.AND TECH.

PANTNAGAR-263145, UTTARANCHAL, INDIA

e-mail: simran_gill@rediffmail.com, kkdube@yahoo.com

Received May 5, 2004; revised version November 19, 2004. 